\title{
Left ventricular thrombi after simultaneous thrombotic occlusions of multiple coronary arteries presenting acute myocardial infarction: need anticoagulant?
}

Hyun Joong Kim, Kyung Jin Choe, Woo-Dae Bang, Sang Jin Ha, Sang-Yong Yoo, and Sangsig Cheong

Division of Cardiology, Department of Internal Medicine, Gangneung Asan Hospital, University of Ulsan College of Medicine, Gangneung, Korea

Received: October 29, 2015 Revised : November 5, 2015 Accepted: November 5, 2015

\author{
Correspondence to \\ Sang Jin Ha, M.D. \\ Division of Cardiology, \\ Department of Internal \\ Medicine, Gangneung Asan \\ Hospital, University of Ulsan \\ College of Medicine, 38 \\ Bangdong-gil, Gangneung 25440, \\ Korea \\ Tel: +82-33-610-3121 \\ Fax: +82-33-641-8130 \\ E-mail: hippoha71@daum.net
}

\section{To the Editor,}

ST-segment elevation myocardial infarction (STEMI) caused by simultaneous thrombotic occlusions of more than one coronary artery, which presents a clinically severe state, is rare. Multiple coronary thromboses arise secondary to identifiable causes (e.g., coronary vasospasm and cocaine abuse) [1], but many cases have no identifiable etiology. Another important issue about post-infarction complications such as left ventricular (LV) thrombi is the absence of guidelines about whether anti-thrombotic strategies, including administration of vitamin $\mathrm{K}$ antagonists, are needed in patients without risks of atrial fibrillation and hypercoagulable status and without LV thrombi on transthoracic echocardiography during hospitalization. Herein, we report a case of LV thrombi after simultaneous thrombotic occlusions of multiple coronary arteries presenting with STEMI with preserved left ventricular ejection fraction (LVEF).

A 57-year-old male patient with histories of current smoking and medication for hypertension as coronary artery risk factors visited our emergency department with a complaint of prolonged chest pain in the early morning. The chest pain persisted for about an hour until hospital admission. The levels of cardiac enzymes were elevated as follows: troponin I $6.64 \mathrm{ng} / \mathrm{mL}$, creatine kinase MB (CK-MB) $61.7 \mathrm{ng} / \mathrm{mL}$, and CK 996 U/L. His vital signs were as follows: initial blood pressure 141/84 $\mathrm{mmHg}$ and pulse rate 72 beats per minute. Electrocardiography demonstrated pathologic Q waves at leads II, III, and aVF, and ST-segment elevation at V3-6 (Fig. 1A). Continuous intravenous infusions of heparin and nitroglycerin were started with aspirin 200 $\mathrm{mg}$, clopidogrel $300 \mathrm{mg}$, and rosuvastatin $20 \mathrm{mg}$. He did not complain of dyspnea, and his auscultation showed no crackle. Chest radiography revealed no evidence of pulmonary edema. Initial echocardiography revealed an LVEF of $50 \%$ with hypokinesia at the apex, lowmid anterior wall, and inferior wall.

A clinical diagnosis of STEMI was made. We performed coronary angiography (CAG). By using CAG (Fig. 1B), complete occlusions were visualized at both the distal left anterior descending (LAD) coronary artery and obtuse marginal artery of the left circumflex (LCx) coronary artery. After intracoronary injection of abciximab as an upstream use of glycoprotein IIb/IIIa, we performed thrombosuction with Thrombuster II 


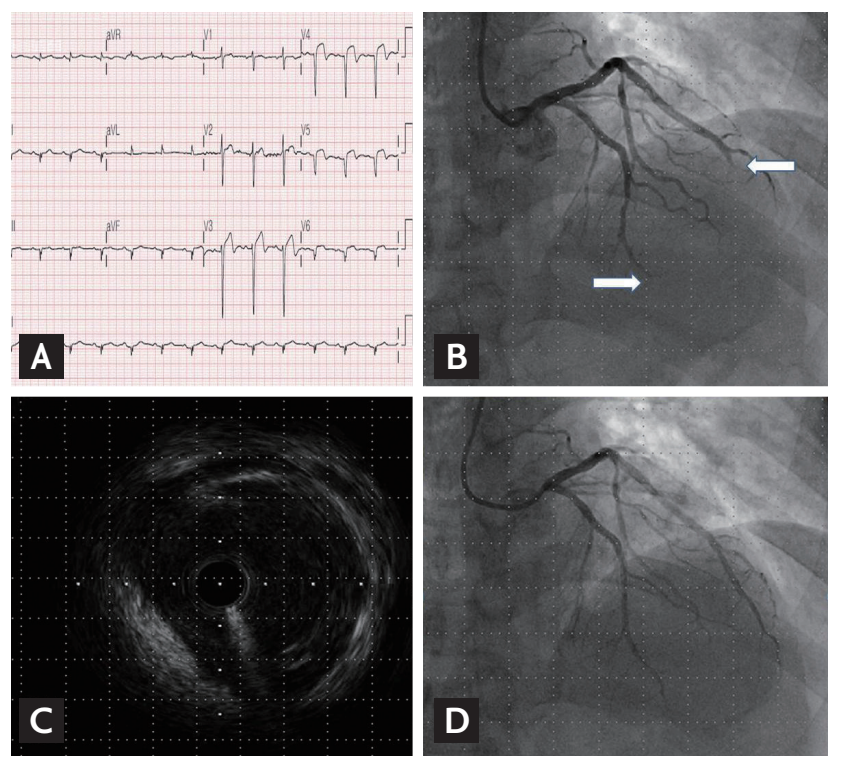

Figure 1. (A) Electrocardiogram demonstrating pathologic $\mathrm{Q}$ waves at leads 11,111 , and $\mathrm{aVF}$, and ST-segment elevation at V3-6. (B) Coronary angiogram showing complete occlusions (arrows) of both the distal left anterior descending coronary artery and obtuse marginal artery of the left circumflex coronary artery. (C) Intravascular ultrasonographic image showing minimal plaques on the left main artery but no evidence of plaque rupture. (D) Coronary angiography obtained after intracoronary injection of abciximab and thrombosuction, showing thrombolysis in the myocardial infarction 2 flow in both coronary arteries.

(Kaneka Corp., Osaka, Japan) at both lesions twice. After thrombosuction, thrombolysis in the myocardial infarction (TIMI) 2 flow was observed at both coronary arteries. To visualize plaques in both coronary arteries, we performed intravascular ultrasonography (IVUS; Boston Scientific, Marlborough, MA, USA). The IVUS revealed minimal plaques on the left main artery but no evidence of plaque rupture (Fig. 1C). After confirmation of TIMI 3 flow, the procedures were finished (Fig. 1D).

Additional 48-hour continuous heparin infusion was administered. For the evaluation of the etiology of the simultaneous thrombus in the LAD and LCx arteries, we performed Holter monitoring for several days and blood tests for the evaluation of hypercoagulable states such as antiphospholipid antibody, homocysteine, proteins $\mathrm{C}$ and $\mathrm{S}$, and factor F Leiden. However, no evidence of hypercoagulable state was found in the blood tests described earlier or atrial fibrillation on Holter examination. On hospital day 7 , the patient underwent ergonovine stress echocardiography for evaluation of vasospasm, which yielded a negative result. Then, he
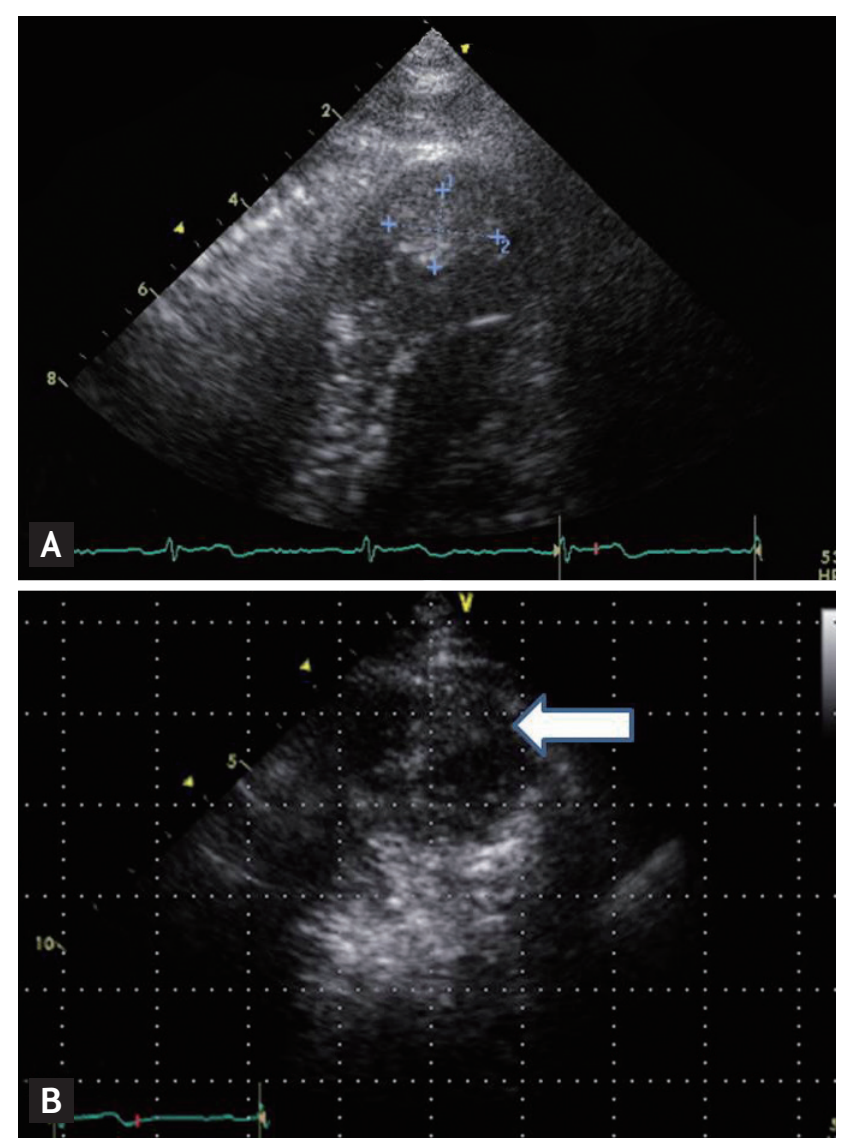

Figure 2. Follow-up transthoracic echocardiogram showing a $1.7 \times 1.2-\mathrm{cm}$ echogenic mass suggestive of left ventricular thrombus. (A) Apical four chamber view. (B) Apical level of parasternal short axis view (arrow).

was discharged from the hospital, with the following medications: aspirin $100 \mathrm{mg}$, clopidogrel $75 \mathrm{mg}$, atorvastatin $20 \mathrm{mg}$, bisoprolol $2.5 \mathrm{mg}$, olmesartan $20 \mathrm{mg}$, and long-acting nitrate. After 1 month, we performed follow-up echocardiography. We found no interval change in regional wall motion abnormality and LV systolic function but found newly developed LV thrombi in the LV apex (Fig. 2). We decided to replace aspirin with warfarin for the management of LV thrombi. He had no cardiac events during the 6-month outpatient follow-up.

A previous single-center study reported simultaneous thrombotic occlusions of more than one coronary artery in approximately $4.8 \%$ of cases of primary percutaneous coronary intervention (PCI) [2]. LV thrombus was one of the post-infarction complications. In Autologous Stem cell Transplantation in Acute Myocardial Infarction trial, among 100 patients who survived from anterior myocardial infarction (MI) with moderate LV dys- 
function (mean LVEF, 45\%), $15 \mathrm{LV}$ thrombi occurred [3]. Among patients with acute MI, the major predictors of LV thrombi were infarct location and size. The patients with large anterior MIs are at the highest risk of developing LV thrombi $[4,5]$. However, whether chronic anticoagulation therapy with vitamin $\mathrm{K}$ antagonist lowers the risk of future cardiovascular events after acute coronary syndrome remains unclear, and triple antithrombotic therapy has not been directly compared with dual antiplatelet therapy (DAPT) in MI patients at risk of LV thrombi. Moreover, the potential benefit of vitamin $\mathrm{K}$ antagonist treatment in combination with DAPT may not overbalance the increased bleeding risk. This calls for a large randomized trial to be implemented to determine whether anticoagulation treatment prevents embolic complications caused by LV thrombi in patients with acute MI treated with primary PCI.

The present case had the following distinct features: despite of simultaneous thrombotic occlusions of more than one coronary artery, completely preserved vital signs and no definite symptom of heart failure without any complications during hospitalization. By contrast, most cases of multiple coronary thromboses are clinically severe and have a high incidence of cardiogenic shock or ventricular arrhythmias. CAG demonstrated little progression of atherosclerosis; thus, revascularization was performed completely only by thrombosuction without coronary angioplasty. However, his risk of recurrent acute coronary syndrome increased after undergoing PCI or coronary artery bypass grafting. Thus, proper medication and close follow-up were necessary. More importantly, LV thrombi developed 1 month after in our case, which had a relatively small infarct size. Clinicians face an obstacle in deciding whether patients who have minimal plaques, no atrial fibrillation, and relatively preserved LV systolic function (LVEF, 50\%) require initial anticoagulation. Ongoing trials can provide the answers on whether anticoagulation should be performed in patients with acute MI who are at risk of LV thrombi.
In conclusion, the incidence of simultaneous thrombotic occlusions of multiple coronary arteries presenting with STEMI is thought to be low but might have been underestimated. The optimal antithrombotic strategy remains unknown; thus, large studies are needed to investigate treatment strategies and evaluations.

Keywords: Acute myocardial infarction; Multiple coronary arteries occlusion; Left ventricular thrombi

\section{Conflict of interest}

No potential conflict of interest relevant to this article was reported.

\section{REFERENCES}

1. Cheitlin MD, McAllister HA, de Castro CM. Myocardial infarction without atherosclerosis. JAMA 1975;231:951-959.

2. Pollak PM, Parikh SV, Kizilgul M, Keeley EC. Multiple culprit arteries in patients with ST segment elevation myocardial infarction referred for primary percutaneous coronary intervention. Am J Cardiol 2009;104:619-623.

3. Solheim S, Seljeflot I, Lunde K, et al. Frequency of left ventricular thrombus in patients with anterior wall acute myocardial infarction treated with percutaneous coronary intervention and dual antiplatelet therapy. Am J Cardiol 2010;106:1197-1200.

4. Chiarella F, Santoro E, Domenicucci S, Maggioni A, Vecchio C. Predischarge two-dimensional echocardiographic evaluation of left ventricular thrombosis after acute myocardial infarction in the GISSI-3 study. Am J Cardiol 1998;81:822-827.

5. Nayak D, Aronow WS, Sukhija R, McClung JA, Monsen CE, Belkin RN. Comparison of frequency of left ventricular thrombi in patients with anterior wall versus non-anterior wall acute myocardial infarction treated with antithrombotic and antiplatelet therapy with or without coronary revascularization. Am J Cardiol 2004;93:15291530 . 